\title{
In-Situ Study of Non-Equilibrium Charge Carriers' Behavior under Ultra-Short Pulsed Electrons Irradiation in Silicon Crystal
}

\author{
Hrant N. Yeritsyan1", Aram A. Sahakyan1, Norair E. Grigoryan1, Vachagan V. Harutyunyan1, \\ Vika V. Arzumanyan', Vasili M. Tsakanov², Bagrat A. Grigoryan², Gayane A. Amatuni²
}

${ }^{1}$ A. Alikhanyan National Science Laboratory (Yerevan Physics Institute), Department of Applied Physics, Yerevan, Armenia

${ }^{2}$ CANDLE Synchrotron Research Institute, Yerevan, Armenia

Email: ^grant@yerphi.am

How to cite this paper: Yeritsyan, H.N., Sahakyan, A.A., Grigoryan, N.E., Harutyunyan, V.V., Arzumanyan, V.V., Tsakanov, V.M., Grigoryan, B.A. and Amatuni, G.A. (2019) In-Situ Study of Non-Equilibrium Charge Carriers' Behavior under Ultra-Short Pulsed Electrons Irradiation in Silicon Crystal. Journal of Modern Physics, 10, 1125-1133.

https://doi.org/10.4236/jmp.2019.109072

Received: June 25, 2019

Accepted: August 3, 2019

Published: August 6, 2019

Copyright () 2019 by author(s) and Scientific Research Publishing Inc. This work is licensed under the Creative Commons Attribution International License (CC BY 4.0).

http://creativecommons.org/licenses/by/4.0/

(c) (i) Open Access

\begin{abstract}
The recombination processes for charge carriers have been studied in $n$-type silicon crystals which were irradiated by pico-second duration pulse electrons with energy of $3.5 \mathrm{MeV}$ (ultrafast irradiation), and maximum dose of $3.3 \times$ $10^{13} \mathrm{el} / \mathrm{cm}^{2}$. In-situ measurements were carried out under artificial conditions simulating natural environment (space, semiconductor detectors, etc.). The observed phenomena were investigated experimentally in-situ using a high-speed oscilloscope equipped with a special preamplifier. Following irradiation to particular doses, some peculiarities of the recovery time of the semiconductor equilibrium condition ("characteristic time"), were obtained. Thus, it was found that the value of the "characteristic time" differs by an order of magnitude from the lifetime of the non-equilibrium (minority) charge carrier measured in an ex-situ regime. However, their behavior, as a function of irradiation dose, is similar and decreases with dose increase. Investigations of the dependencies of electro-physical parameters on irradiation dose, using Hall effect measurements, showed that at particular doses the radiation defects thus created, have an insignificant influence on the concentration of the charge carriers, but change their scattering properties appreciably, which affects the time parameters for the recombination of the semiconductor charge carriers. This investigation uses a novel approach to solid-state radiation physics, where in situ measurements were conducted in addition to conventional pre- and post-irradiation.
\end{abstract}

\section{Keywords}

Silicon Crystal, Irradiation, Recombination, Non-Equilibrium State, 


\section{Introduction}

The study of recombination processes in irradiated semiconductors as a nonequilibrium state, mainly in silicon crystals, is of significance because it determines the behavior of the electro-physical parameters for these materials: conductivity, concentration and mobility of charge carriers, and a characteristic property, which is the lifetime of the minority charge carriers. The main factors that prevent the achievement of an equilibrium state under conditions of irradiation are the energetic parameters of impurity redistributions and vacancy-interstitials, both as initially present, and those which are created during irradiation [1] [2] [3] [4] [5]. These radiation defects are often responsible for the degradation of devices that contain silicon crystals, and even in the absence of degradation, the performance of a device can be impeded by the presence of defects. Those defects which have energy levels within the bandgap can act as recombination centers, and affect the carrier collection within a device. These effects can sometimes be used to advantage, since the frequency characteristics of devices may be increased by the presence of a high concentration of defects, and thus, the optical generation of $\mathrm{THz}$ pulses is possible, in semiconductors with sufficiently large defect densities that the carrier lifetimes are as short as a few picoseconds [4]. The radiation-induced changes in properties of the silicon used in the tracking detectors, that are employed in different areas of science and technology, mainly occur as a result of the formation and interaction of radiation defects with impurities, and these fundamentally influence the electrical parameters of the detectors, their efficiency [6] [7].

The formation of a non-equilibrium state in irradiated host crystals is well known [7] [8] [9]; however only a few initial investigations have been made using radiation applied at very short pulse durations [10] [11] [12], whereas such investigations are highly demanded both in theory and practice, particularly in space applications. The concept of a non-equilibrium state provides a basis for reinterpretation of the following radiation effects: formation of stable state of the crystal properties; differences in the radiation, thermal and time stability of materials with different impurity contents; injection of impurity atoms into the positions where intrinsic interstitial atoms are trapped; and thermal processes that occur in irradiated crystals: the latter is not feasible in the current study, because the thermal processes are not activated on the short timescale of pico-second pulse irradiation [11].

The influence of high-density pulse irradiation in current study-which results in an intensive ionization of semiconductors, with the generation of additional non-equilibrium charge carriers-is significant, since it may give rise to large non-stationary currents and electrostatic forces in electronic devices, and hence, damage them. 
In the present paper we report the results from some experiments in which silicon crystals were irradiated using pico-second duration pulse electrons, to create a non-equilibrium state. This state is characterized by recombination processes, which take place in semiconductor materials and act as trapping centers for charge carriers, and are determined by the carrier lifetimes (often those of minority carriers) at the centers. In silicon, which is an indirect-bandgap semiconductor, the recombination centers are efficient when the radiation defect level is close to the middle of the bandgap, since it has a large capture cross-section for holes and electrons [13] [14]. Hence, it can be concluded that the lifetime of the minority charge carriers $(\tau)$ is the most useful parameter for the characterization of non-equilibrium processes in irradiated semiconductors, in particular, crystalline silicon. Indeed, $\tau$ is very sensitive to the effect of irradiation, and changes by an order of magnitude at low radiation doses, although the specific resistivity shows practically no change. Below the results of measurements and discussion are given.

\section{Experimental Procedure and Results}

Although many energy levels are created in the forbidden gap of crystalline silicon when it is irradiated, the behavior of minority charge carriers' lifetime $(\tau)$ is determined by just one or two such levels. For example, the A-centre (Oxygen atom with a complex vacancy) is the dominant radiation defect formed in silicon crystals that have been grown using the Czochralski method, which is located in the upper half $\left(\mathrm{E}_{\mathrm{c}}-0.17 \mathrm{eV}\right)$ of the bandgap and acts as an acceptor level in $n$-Si. The E-centre (Phosphorus atom with a complex vacancy) is the most dominant recombination centre in silicon that has been grown using the floating-zone method (doping with Phosphorus), which is located at the $\mathrm{E}_{\mathrm{c}}-0.41 \mathrm{eV}$ level of the bandgap and also acts as an acceptor level in $n$-Si [1].

In previous studies, we have shown that the application of pico-second duration pulse irradiation mainly generates A-centre type defects (in the form of clusters) in $n$-Si crystals [12], and in the following, we describe measurements of this center specifically. In the current paper a determination is made of the conductivity, concentration of charge carriers, their mobility, the lifetime of the minority charge carriers, in irradiated samples, and a detailed analysis of the results was made in order to elucidate the nature of the recombination centers. A standard Hall effect method [15] and a lifetime of the minority charge carriers' recombination method, based on HF (high-frequency) light-absorption [16] are used. All measurements were carried out both before and after irradiation at room temperatures.

The irradiation was performed using the linear electron accelerator AREAL at the CANDLE organization [11]. Time dependent measurements of the resistivity of the silicon crystals were made, in-situ, during irradiation, and the occurrence of recombination was observed on oscilloscope. The use of a stabilized current source and a high-speed oscilloscope, fitted with a preamplifier, enabled the ob- 
servation of the dynamics of changes in the resistivity of the sample, with an accuracy of $\pm 0.5 \mu \mathrm{s}$ on the distance of $20 \mathrm{~m}$. The parameters of the electron beam were: electron energy $3.5 \mathrm{MeV}$, pulse duration $4 \times 10^{-13} \mathrm{~s}$, charge 50 pico-Coulomb and the frequency was $12 \mathrm{~Hz}$, which corresponds to an intensity of $10^{9} \mathrm{el} / \mathrm{cm}^{2} \cdot \mathrm{s}$. The $n$-type silicon single crystal samples were of dimension, $10 \mathrm{~mm}$ $\times 4 \mathrm{~mm} \times 1 \mathrm{~mm}$, and had a dual-cross form and a specific resistance of 120 $\Omega \cdot \mathrm{cm}$. The irradiation scheme is presented in Figure 1. Following the irradiation, the main parameters of $n$-Si were again measured.

The lifetime of the minority charge carriers $(\tau)$ in $\mathrm{Si}$ was determined nondestructive by measuring the decay time of the signal of the recombination process of nonequilibrium charge carriers in a semiconductor. Non-equilibrium charge carriers are generated by illumination of a sample with a radiation wavelength of $1.05 \mu \mathrm{m}$, the decay process of which is detected by microwave absorption at a frequency of $10 \mathrm{GHz}$, which is recorded on an oscilloscope. The accuracy of the measurement is $\pm 10 \%$ [16]. A significant aspect of these experiments is that all parameters were measured on the same sample, for both in-situ and $e x$-situ regimes.

Experimental results are given in Figures 2-4, in the form of oscilloscope pictures and dose dependences of the electro-physical parameters of the $n$-Si crystal samples. The change of resistivity of the silicon sample under in-situ irradiation to different doses $R=R(t)$ is presented in Figure 2 by the oscilloscope pictures (OP). The behavior of $R=R(t)$ during pico-second pulse irradiation was characterized by a particular time, $\Delta t$, which represents the recovery time of the sample's resistivity following the irradiation pulse (see Figure 2(a)). It is important to note that this OP corresponds to the starting point of the irradiation process, which is about 10 seconds. It is obvious from the OP-s presented that $\Delta t$ characterizes the dynamics of the crystal resistivity, and primarily depends on the initial stages of the irradiation (see $\Delta t(D)$ in Figure 3), and the form of the OPs can be seen to vary, along with changes in the resistivity of the crystal.

As the irradiation dose accumulates, the form and parameters of the OPs are substantially changed. It is known that the recombination processes of holes and electrons in silicon crystals are well described by an exponential function. At low irradiation doses, the initial regions of the $R=R(t)$ curves are non-linear (see Figure 2(a) and Figure 2(b)), but as the dose increases, they become nearly linear (Figure 2(c) and Figure 2(d)), which may be due to an increase in the density of radiation defects. From Figure 2 it is clear that exponential relationship applies at the final region of the diagram, and thus it is reasonable to suppose that there is a different mechanism operating here, for the recovery of the resistivity, than exists at the initial stage.

It is apparent from Figure 3 that both the characteristic time $\Delta t$, and the lifetime of the minority charge carriers, $\tau$, decrease as the irradiation dose accumulates, and the $\Delta t(D)$ curve has some peculiarity. The value of $\Delta t$ was found to decrease following each irradiation dose, and as a result of being stored 


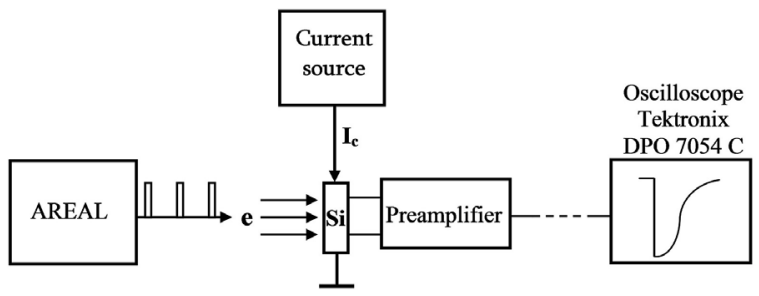

Figure 1. Schematic diagram of the sample irradiation and observation of resistivity change at in-situ process.
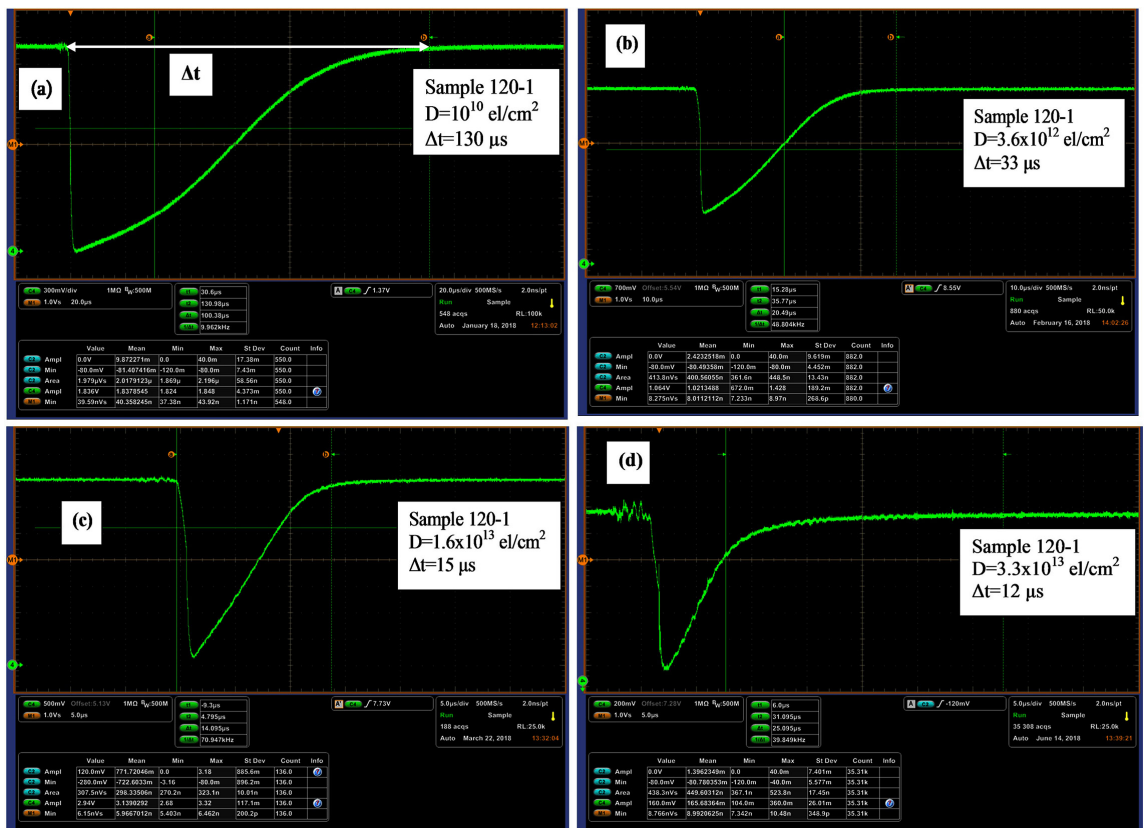

Figure 2. Oscilloscope pictures showing the dynamics of changes in the resistivity of a crystalline silicon sample, measured in-situ under the influence of picoseconds electron pulse irradiation: (a) at the initial stage of irradiation, (b) after irradiation to a dose of 3.6 $\times 10^{12} \mathrm{el} / \mathrm{cm}^{2},(\mathrm{c})$ after irradiation to a dose of $1.1 \times 10^{13} \mathrm{el} / \mathrm{cm}^{2},(\mathrm{~d})$ after irradiation to a dose of $3.3 \times 10^{13} \mathrm{el} / \mathrm{cm}^{2}$. In all cases the sample temperature was $\mathrm{T}=300 \mathrm{~K}$.

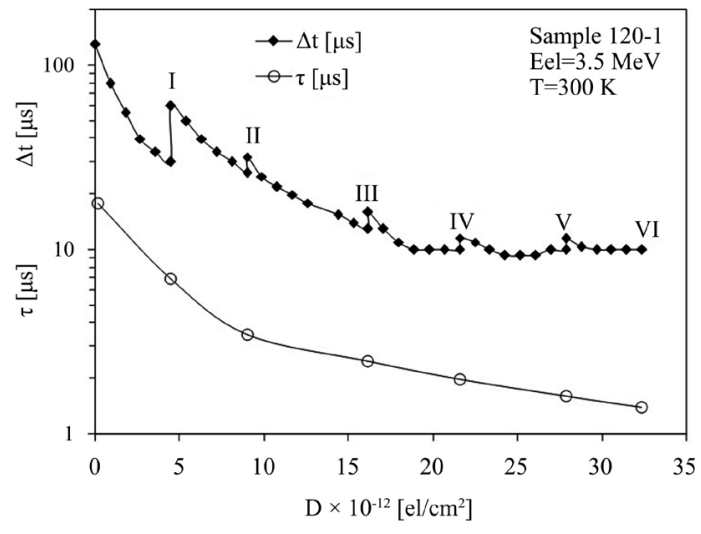

Figure 3. Pico-second electron pulse irradiation dose dependences of the characteristic time $\Delta t(D)$ and the lifetime of the minority charge carriers $\tau(D)$ in crystalline Si. On the $\Delta t(D)$ curve the values of $\Delta t$ are presented in terms of defined stages of irradiation doses, and above the curve the number of stages is shown. 

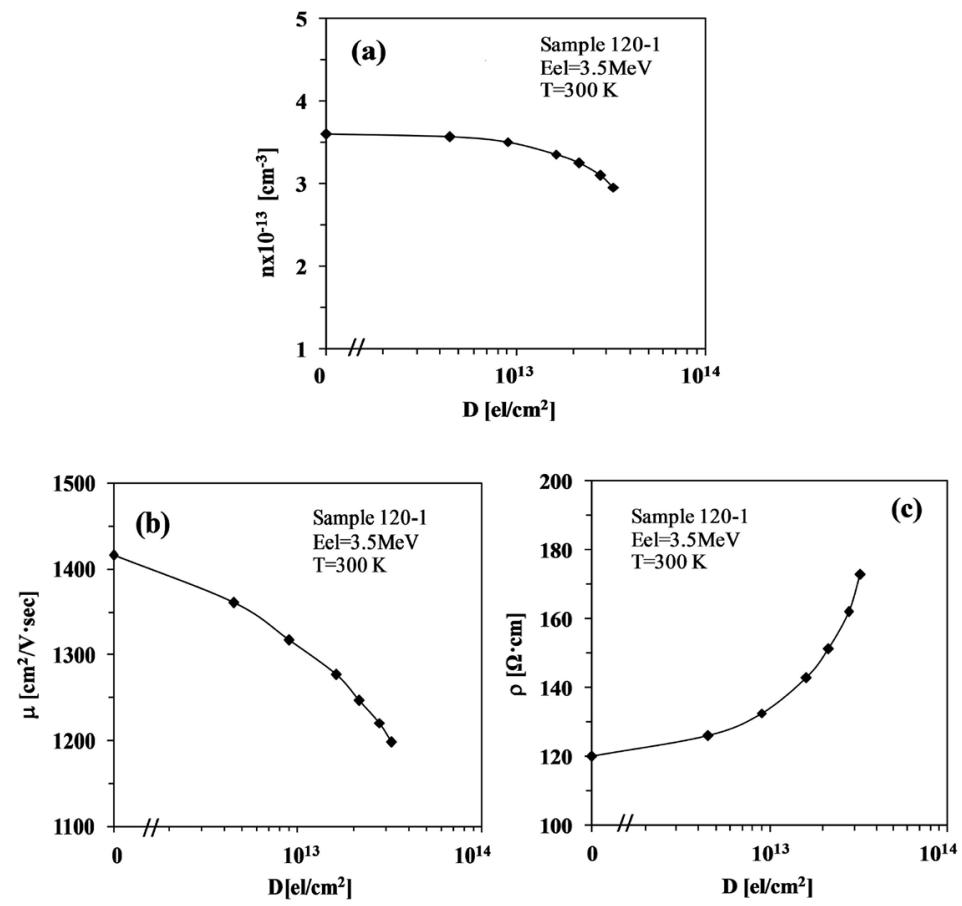

Figure 4. Pico-second electron pulse irradiation dose dependences of: (a) main charge carriers' concentration $-n(D)$, (b) mobility $-\mu(D)$ and (c) specific resistivity- $\rho(D)$ of $n$-Si with initial specific resistivity $120 \Omega \cdot \mathrm{cm}$.

for one month (stage duration) it increased significantly above the initial value. As a result of further irradiation and storage the peak decreases, and finally almost disappears as the degree of irradiation increases.

The dose dependencies of the electro-physical parameters of samples measured at room temperatures both before and after irradiation are presented in Figure 4. It can be seen that the concentration of the main charge carriers, $n$, at initial irradiation doses are practically unchanged after two stags, while the mobility $\mu(D)$ and specific resistivity $\rho(D)$ are changed significantly. Hence, at low irradiation doses, the mobility of the main charge carriers is more sensitive to the ultrafast irradiation.

\section{Discussion}

The transformations of defect complexes may occur by direct interaction with vacancies, interstitials or impurities. Since the impurity concentrations often are higher than the saturation limit, a redistribution of the concentrations of the impurities may occur in sites and interstitial positions, and interact with point crystal defects to form defect complexes such as A- or E-centers, or other defects that involve the participation of the impurity atoms. Hence, the displacement of impurity atoms from the lattice sites in interstitial positions will occur in any crystal with impurity concentrations higher than at the non-equilibrium state and this process will become more important for higher concentrations of impurities and will be dependent on the conditions (e.g. dose) of the irradiation. 
We offer an explanation of the observed phenomena, which relate to the time-scales of the recombination processes, in terms of an annealing of meta-stable radiation defects at room temperatures, and the establishment of an equilibrium condition of the crystal with stable defects, i.e. "steady state" of the silicon crystal. The behavior of lifetime $\tau(D)$ in Figure 3 corresponds with results reported in the literature (without any peculiarity) and is explained by a decrease in $\tau$ as the concentration of radiation defects increases, which are created by irradiation, as a result of a more effective trapping of minority charge carriers [10] [13]. The analogous dependences of both $\Delta t(D)$ and $\tau(D)$ emphasize that a process occurs involving the recombination of charge carriers, in result of irradiation, which achieves "steady state" after defined irradiation dose (in this case, $3.3 \times 10^{13} \mathrm{el} / \mathrm{cm}^{2}$ ). It is possible that different factors may influence the outcome of illumination of a sample with radiation of a $1.05 \mu \mathrm{m}$ wavelength, in the case of $\tau$ measurements, while it is the $\Delta t$ parameter which is being determined in-situ during irradiation by electrons with energy of $3.5 \mathrm{MeV}$, i.e. there are different crystal excitation mechanisms in operation. It is possible that the pico-second duration pulse irradiation may create some plasma effects in the crystals, and thus influence their properties as a result of a significantly high density of radiation defects, a subject which we intend to explore in additional investigations.

It has been mentioned in the literature [17] [18] [19] that a divacancy type radiation defect is the main trap for free charge carriers, and its formation in irradiated $\mathrm{Si}$ crystals leads to an increase in the resistivity of the material which is an amphoteric center and has three energy levels in the Si bandgap. However, in our experiments, despite the fact that the resistivity increases during irradiation, three energy levels were not observed and we consider that A-centers are the main recombination centers for minority charge carriers. In any case, it is more likely that divacancy type radiation defects will be created in silicon, by irradiation with very high energy electrons (higher than $10 \mathrm{MeV}$ ), neutrons or protons [18] [19] [20].

\section{Conclusions}

Thus, the in-situ observation of the behavior of the electro-conductivity for silicon crystals under conditions of pico-second duration pulse electron irradiation with an energy of $3.5 \mathrm{MeV}$, suggests that the transition of the crystal from a non-equilibrium state to a "steady state", takes place by a process involving the recombination of charge carriers, and has some particular characteristic which is a consequence of their interaction times.

1) The value of the "characteristic time", $\Delta t(D)$, observed under in-situ condition is 10 times higher than the lifetime of the minority charge carriers $\tau(D)$, measured at $e x$-situ, despite the fact that both decrease with increasing irradiation dose.

2) Meta-stable defects were observed, which can be annealed at room temper- 
ature, and the irradiation dose at which this phenomenon disappeared $(3.3 \times$ $10^{13} \mathrm{el} / \mathrm{cm}^{2}$ ), i.e. when a "steady state" occurs, was determined.

3) It was shown that at low doses of pico-second pulse irradiation, the specific resistivity change of silicon is determined by the mobility of the charge carriers, rather than by their concentration.

4) Detailed analysis of the OPs demonstrated that there is additional process that influences the relaxation during the recovery of the equilibrium state under irradiation of crystalline silicon.

5) Future investigations are planned with theoretical interpretation to elucidate the physical nature of the $\Delta t$ parameter because it may reveal behavior of semiconductor materials and devices operating in the real processes.

\section{Acknowledgements}

The authors acknowledge support of this work by the State Committee of Science of the Ministry of Education and Science Republic of Armenia, in the framework of the research project grant No. 17A-1C002. The authors are thankful to Christopher Rhodes for useful discussion.

\section{Conflicts of Interest}

The authors declare no conflicts of interest regarding the publication of this paper.

\section{References}

[1] Hallen, A., Keskitalo, N., Masszi, F. and Nagl, V. (1996) Journal of Applied Physics, 79, 3906. https://doi.org/10.1063/1.361816

[2] Emtsev, V.V. and Mashovets, T.V. (1981) Impurities and Point Defects in Semiconductors. Radio I Svyaz, Moscow, 248 p. (In Russian)

[3] Takakura, K., Ohyama, H., Murakawa, H., Yoshida, T., et al. (2004) Journal of Applied Physics, 27, 133. https://doi.org/10.1051/epjap:2004058

[4] Krotkus, A. (2010) Journal of Physics D: Applied Physics, 43, Article ID: 273001. https://doi.org/10.1088/0022-3727/43/27/273001

[5] Mukashev, N., Abdullin, K.A., et al. (2000) Rus. Usp. Phys. Nauk, 170, 143.

[6] Radu, R., Pintilie, I., Nestor, L.C., et al. (2015) Journal of Applied Physics, 117, Article ID: 164503. https://doi.org/10.1063/1.4918924

[7] Lee, Y.H., Brosious, P.R. and Corbett, J.W. (1974) Physical Review B, 9, 4351. https://doi.org/10.1103/PhysRevB.9.4351

[8] Smirnov, L.S., Bolotov, V.V. and Vasilyev, A.V. (1979) The Effect of Semiconductor Crystals Unequilibrium State on Radiation Processes. International Conference on Radiation Physics of Semiconductors and Related Materials, Tbilisi, 13-19 September 1979, 334-337.

[9] Makarov, V.A. and Ukhin, N.A. (1979) The Influence of Particle Energy on the Efficiency of Recombination Rate Change in Si and Ge. International Conference on Radiation Physics of Semiconductors and Related Materials, Tbilisi, 13-19 September 1979, 410-413. 
[10] Dvurechenski, A.V., Kashnikov, B.P. and Smirnov, L.S. (1979) Defects in Silicon Irradiated with High Current Density Electron Pulses. International Conference on Radiation Physics of Semiconductors and Related Materials, Tbilisi, 13-19 September 1979, 305-307.

[11] Yeritsyan, H.N., Sahakyan, A.A., Grigoryan, N.E., Harutyunyan, V.V., Tsakanov, V.M., Grigoryan, B.A., Amatuni, G.A., Petrosyan, V.H., Khachatryan, A.A. and Rhodes, C.J. (2018) Journal of Electronic Materials, 47, 4010-4015.

https://doi.org/10.1007/s11664-018-6286-6

[12] Yeritsyan, H.N., Sahakyan, A.A., Grigoryan, N.E., Harutyunyan, V.V., Khachatryan, A.A., Grigoryan, B.A., Avagyan, V.S., Amatuni, G.A. and Vardanyan, A.S. (2016) Journal of Modern Physics, 7, 1413. https://doi.org/10.4236/jmp.2016.712128

[13] Konopleva, R.F. and Ostroumov, V.I. (1975) The Interaction of High Energy Charged Particles with Silicon and Germanium. Atom-Edition, Moscow, 128 p.

[14] Schroder, K. (1997) IEEE Transactions on Electron Devices, 44, 160-170. https://doi.org/10.1109/16.554806

[15] Kauppinen, H., Korbel, C. and Skog, C. (1997) Physical Review B, 55, 9598. https://doi.org/10.1103/PhysRevB.55.9598

[16] Eremin, V., Ivanov, A., et al. (1999) Nuclear Instruments and Methods in Physics Research Section A, 426, 120. https://doi.org/10.1016/S0168-9002(98)01479-X

[17] Poiler, V.A., Dannefaer, S. and Schiettekatte, F. (2003) Nuclear Instruments and Methods in Physics Research Section B, 206, 85. https://doi.org/10.1016/S0168-583X(03)00686-4

[18] Kozlovski, V.V. and Kozlov, V.A. (2001) Semiconductors, 35, 735. https://doi.org/10.1134/1.1385708

[19] Wondrak, W., Bethke, K. and Silber, D.G. (1987) Journal of Applied Physics, 62, 3464. https://doi.org/10.1063/1.339289

[20] Lang, D.V. (1977) Point Radiation Defects in Semiconductors. Proceedings International Conference on Radiation Defects in Semiconductors, Dubrovnik, Institute of Phys. Conf. Ser. 31, 70-94. 